\title{
Design of Porous Organic Polymer based Promising Molecular Nanoarchitecture in Advanced Heterogeneous Catalysis
}

\author{
Sudipta K. Kundu², Ramana Singuru ${ }^{1}$, Asim Bhaumik², Benjaram Mahipal Reddy ${ }^{1}$, John Mondal ${ }^{1 *}$ \\ ${ }^{1}$ Inorganic and Physical Chemistry Division, CSIR-Indian Institute of Chemical Technology, Uppal Road, Hyderabad, 500007 \\ ${ }^{2}$ Department of Materials Science, Indian Association for the Cultivation of Science, Kolkata, 700032, India
}

Received: January 23, 2016; Accepted: February 17, 2016; Published: March 08, 2016

*Corresponding author: John Mondal, Inorganic and Physical Chemistry Division, CSIR-Indian Institute of Chemical Technology, Uppal Road, Hyderabad, 500007, India.E-mail: johncuchem@gmail.com; johnmondal@iict.res.in

\begin{abstract}
Porous organic polymers (POPs) having very high surface areas, tunable pore volumes, excellent stabilities and controllable chemical composition have recently emerged a growing research interest as a most promising molecular nanoarchitecture for binding of metal or metal oxide nanoparticles (NPs) in the development of new advanced heterogeneous catalysts. Here in this featured article, we have focused on development of various kinds of POPs and immobilization of NPs in the framework of these POPs with their prospective employment as heterogeneous nanocatalysts to catalyze several catalytic reactions. These POP-based materials exhibit superior catalytic performance in coupling, hydrogenation and various acid catalyzed reactions with tremendous recyclability and combination of best features of both homogeneous and heterogeneous catalysts.
\end{abstract}

Keywords: Porous Organic Polymers; Heterogeneous Nanocatalysis; Rigid Framework; Heterogeneous Organocatalyst

\section{Introduction}

The synthesis of Porous Organic Polymer (POP) support metal and metal oxides, it has received an increasing attention over the last decade in interdisciplinary research area for the development of new heterogeneous catalysts owing to their high chemical activity and wide-ranging applicability [1,2]. Generally, ultrafine metal NPs are very efficient in catalytic reactions than their bulk metal particles owing to their high surface to volume ratio so that they can provide a large number of available active sites per unit area for reaction substrates. But owing to their high surface energy, aggregation and thermally induced sintering of nanoparticles during catalytic reactions accompanied by a diminishment in catalytic activity always take place [3]. However, numerous obdurate problems including usual detachment of metal nanoparticles from the support and general tendency to grow or aggregate during the catalytic process are still associated which hamper the progress of this research area. To address these above mentioned problems, various molecular architecture for encapsulation or fabrication of NPs including mesoporous silica, zeolite, metal organic framework (MOFs) have been developed in recent years [4,5]. But among them Porous Organic Polymers (POPs) have been emerged a incredible degree of awareness as a most promising molecular nanoarchitecture for immobilization, fabrication, encapsulation followed by stabilization of ultrafine metal NPs because of their huge surface area with well-defined pore size distribution, high thermally and mechanically stable covalent bonds, low skeleton density, and presence of hydrophobic components in their framework which exhibits high catalytic activities and also high selectivity [6,7]. Spatial control of catalytically active sites in porous organic materials can be tuned and modified through various choice of organic building blocks and synthetic diversity, which provides them potential ability for catalysis $[8,9]$. Practical application of MOF (Metal Organic Framework) encapsulated NPs in heterogeneous catalysis remains yet unexplored owing to their moisture sensitivity, physiochemical instability in acids or bases and presence of metal and co-ordination bonds $[10,11]$. An additional stability and progressive catalytic activity of metal NPs in Porous Organic Polymer compared with the other catalytic supports is obtained owing to the interaction between organic linkers in POPs and metal NPs that can be readily enhanced by introducing coordination groups into metal precursors. In addition, easy recovery of these heterogeneous catalysts by simple filtration technique after the reaction and tremendous recyclability with negligible lose of catalytic activity have attracted a broad research interest in catalysis community for production of fine chemicals, value-added products in petroleum refining and chemical industry. The past decade has witnessed performance of various metal-catalyzed couplings, including Yamamoto, Suzuki-Miyaura, Sonogashira-Hagihara, Cucatalyzed click reaction and Friedel-Crafts reactions, radical polymerization with AIBN for the preparation of such POPs [12-17]. To date, extensive efforts have been focused in the development of such POPs supported materials with facile fabrication or encapsulation of metal or metal oxide NPs on the external surface as well as in the interior cavity of those respective functionalized POPs. Recent reports in the literature demonstrate that heterogeneous catalysts for morphology-tuned aerobic oxidation [18] hydrogenation [19] coupling [20] cycloaddition reactions, [21] and 
so forth can be developed through the fabrication and immobilization of metal NPs inside the nanoporous channel of POPs. Recently a number of research groups have reported many palladium NPs immobilized in imidazolium-based POPs used in advanced heterogeneous catalysis with high catalytic efficiency and recyclability. Wang, et al. discovered an interesting synthetic strategy for in situ formation of well-dispersed palladium NPs immobilized in imidazolium-based organic ionic polymers (OIP-1 and OIP-2) through Suzuki-Miyaura cross-coupling reaction of 1,3-bis(4-bromophenyl) imidazolium bromide with 1,3,5-tri(4pinacholatoborolanephenyl) benzene in 1,4-dioxane and DMF for hydrogenation of nitroarenes to produce aniline derivatives at 25 ${ }^{\circ} \mathrm{C}$ [19]. They have found that Pd@OIP-2 offers higher catalytic activity and recyclability at least $6^{\text {th }}$ competitive run without significant loss of catalytic activity compared with Pd@OIP-1 with their exposed Pd(111) crystalline facets making a better contact between substrate and catalytic active Pd-sites. They have also developed various palladium N-heterocyclic carbene polymers. They have synthesized poly-imidazolium particles with wireshaped, spherical and ribbon-shaped morphologies by adjusting solvent polarity, solubility, concentration and temperatures. These morphologies remains unaltered after complexation of the particles with $\mathrm{Pd}(\mathrm{OAc})_{2}$ to produce the corresponding ionic polymers containing palladium $\mathrm{N}$-heterocyclic carbene units (NHCs) which showed morphology dependant catalytic activity in heterogeneous Suzuki-Miyaura cross-coupling reaction [22]. Wireshaped palladium-NHC polymer exhibits excellent catalytic activity, probably due to larger contact areas with the reactants of wire-shaped particles. Due to superparamagnetic nature of $\mathrm{Fe}_{3} \mathrm{O}_{4^{\prime}}$ the newly developed material has recently emerged a growing attention as a novel magnetically separable catalyst. Recently, Wang et. al. also synthesized two spherical magnetic core-shell palladium-NHC particles $\left(\mathrm{Fe}_{3} \mathrm{O}_{4} @ \mathrm{PNP} 1\right.$ and $\left.\mathrm{Fe}_{3} \mathrm{O}_{4} @ \mathrm{PNP} 2\right)$ by a one-pot reaction of tripodal imidazolium salts and $\mathrm{Pd}(\mathrm{OAc})_{2}$ in the presence of magnetite nanoparticles [23]. These magnetically separable heterogeneous catalysts display outstanding catalytic activity and recyclability in Suzuki-Miyaura cross-coupling reaction at $25{ }^{\circ} \mathrm{C}$ in $\mathrm{H}_{2} \mathrm{O}$-EtOH mixture. Karimi's group recently developed a new protocol to synthesize another N-heterocyclic carbene-Pd polymers, which was found an efficient precatalyst for cyanation of various aryl halides by using non-toxic cyanic source $\left[\mathrm{K}_{4} \mathrm{Fe}(\mathrm{CN})_{6}\right]$ under mild condition to produce corresponding benzonitrile derivatives [24]. Palladium NPs encapsulated 1,2,3-triazolyl containing porous organic polymers have been described by Wang's group [25]. These well designed CPP-C and CPP-Y polymers have been synthesized through click reaction and Yamamoto coupling reaction, respectively with the corresponding BET surface areas are 156 and $408 \mathrm{~m}^{2} \mathrm{~g}^{-1}$, respectively, and their total pore volumes are 0.19 and $0.25 \mathrm{~cm}^{3} \mathrm{~g}^{-1}$, respectively. Ultrafine palladium NPs are successfully incorporated in the interior cavities of these polymers to synthesize corresponding Pd@ CPP-C and Pd@CPP-Y materials and its application as heterogeneous catalysts in hydrogenation of olefins to alkanes at $25{ }^{\circ} \mathrm{C}$ and $1 \mathrm{~atm}$ of $\mathrm{H}_{2}$ was investigated and high efficiencies and selectivities were achieved. Furthermore, Pd@CPP-Y was reused for at least 7 catalytic runs without obvious activity loss. More re- cently, another two click-based porous organic polymers (CPP-1 and CPP-2) are designed by click reaction of tetrakis-(4-ethynylphenyl) methane with 4,4'-diazidobiphenyl and tetrakis(4azidophenyl)-methane with 4,4'-diethynylbiphenyl, respectively in the presence of copper sulfate and sodium ascorbate with very high BET surface areas 826 and $894 \mathrm{~m}^{2} \mathrm{~g}^{-1}$, respectively [26]. Ultrafine Pd-NPs with dual distributions and no apparent aggregation are successfully confined in the organic skeleton to develop Pd-based nanocatalyst in hydrogenation of nitroarenes at $25{ }^{\circ} \mathrm{C}$ and $1 \mathrm{~atm}$ of $\mathrm{H}_{2}$ in ethanol medium with superior stability, recyclability without loss of catalytic activity and enhancement in activity compared with the palladium on charcoal. Mondal, et al. have reported the synthesis of a three-dimension benzene-benzylamine based porous organic polymer (BBA-1) network with a high BET surface area by adopting a novel strategy through Friedel-Crafts alkylation of benzylamine and benzene by using formaldehyde dimethyl acetal as a cross-linker and anhydrous $\mathrm{FeCl}_{3}$ as a promoter [27]. Ruthenium Nanoparticles (Ru-NPs) anchored BBA-1 POP based materials (Ru-A, Ru-B and Ru-C) have been successfully synthesized through $\mathrm{NaBH}_{4}$, ethylene glycol, and hydrothermal reduction routes. These newly developed materials exhibited excellent catalytic performance in room temperature catalytic transfer hydrogenation of nitroarene with very high conversion, selectivity, improved catalytic activity compared with the conventional catalysts such as Ru@C, Ru@SiO${ }_{2}$, and $\mathrm{Ru} @ \mathrm{TiO}_{2}$ owing to the synergetic effect of nanoconfinement and electron donation offered by the 3D POP network. For the selective aerobic oxidation of hydrocarbons and alcohols to produce more functional compounds including aldehydes, ketones and imines with atmospheric air or molecular oxygen, several heterogeneous catalysts have been developed. New salen-(Mn)-based porous organic polymer (Mn-POP) with BET surface area 836 $\mathrm{m}^{2} \mathrm{~g}^{-1}$, and $3.4 \mathrm{~nm}$ pore dimension has been synthesized by Cao et al. employing palladium catalyzed Sonogashira-Hagihara coupling reaction of 1, 3, 5-triethynylbenzene and Mn-salen complex [28]. This newly designed Mn-POP was utilized as an efficient heterogeneous catalyst for olefin epoxidation with high activity and excellent selectivity to epoxide using $\mathrm{O}_{2}$ as an oxidiser. The catalyst is easily separable and could be recycled at least five consecutive catalytic runs and no considerable drop in conversion and epoxide selectivity is observed during recycling. Morphology-tuned catalytic activity of POPs supported metal oxides has attracted considerable interest in recent time. Zhao et. al. have performed an extensive investigation on shape-controlled catalytic activity for $\mathrm{Mn}_{3} \mathrm{O}_{4}$ nanoparticles with sphere, nanowire and octahedron morphologies fabricated on divinyl benzene-triallyl amine (DVTA) POP in aerobic $\mathrm{sp}^{3} \mathrm{C}-\mathrm{H}$ bond oxidation of aromatic hydrocarbons and alcohols [29]. The synthesis of a new class of divinyl benzene based Porous Organic framework with huge surface area $903 \mathrm{~m}^{2} \mathrm{~g}^{-1}$ and pore volume is $0.472 \mathrm{ccg}^{-1}$ was achieved by Non-aqueous polymerization of a mixture of divinylbenzene and triallyl amine under hydrothermal conditions in presence of Azobisisobutyronitrile (AIBN) as the radical initiator. Moreover, Mn@DVTA-3 nanocatalyst having octahedron morphology exhibited exceptionally higher catalytic activity compared with Mn@DVTA-1 and Mn@DVTA-2 owing to more exposed (101) 


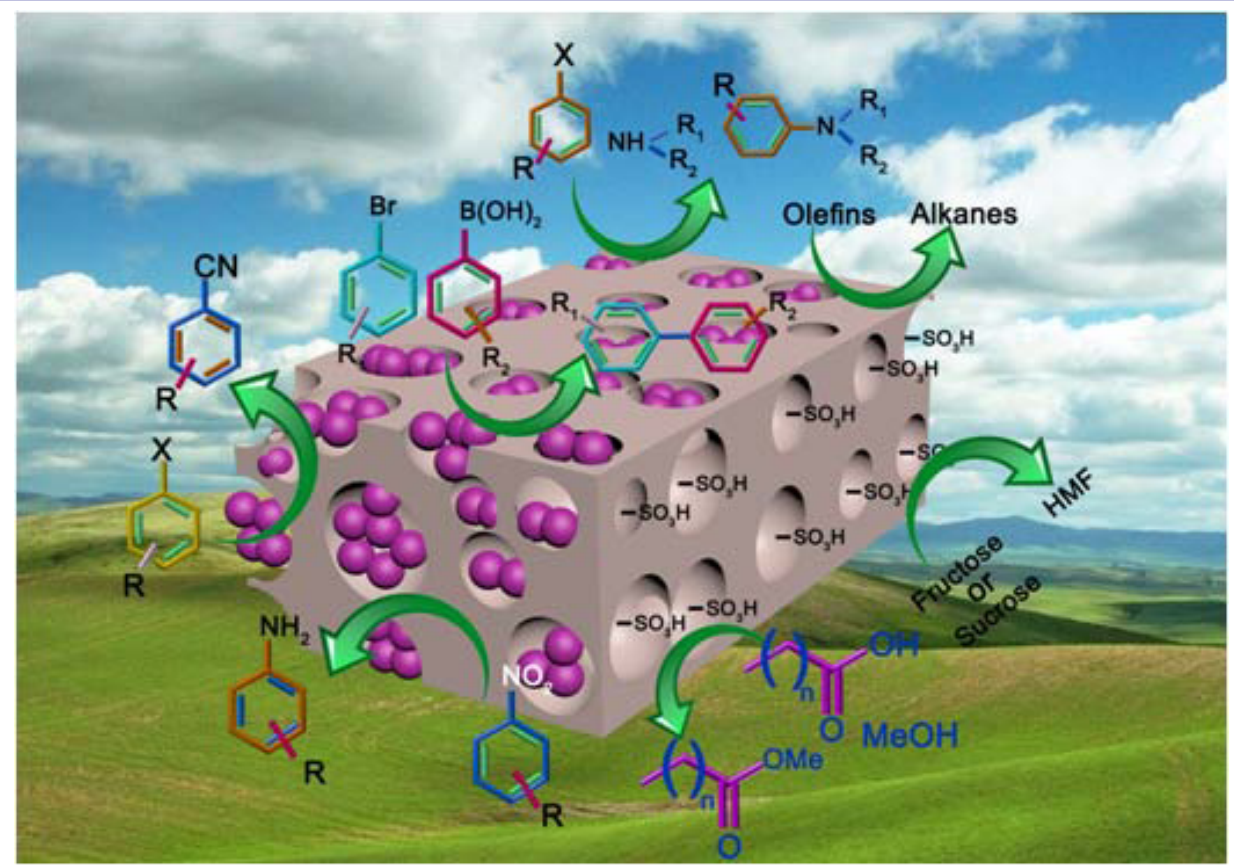

Figure 1: Schematic presentation Porous Organic Polymer (POP) based molecular Nanoarchitecture used in Advanced Heterogeneous Catalysis.

Table 1: Catalytic activity of various porous organic polymer supported metal NPs and acid fuctionalized microporous polymers.

\begin{tabular}{|c|c|c|c|}
\hline Materials & Reaction conditions & Application in catalysis & Reference \\
\hline Pd@0IP-1 and Pd@OIP-2 & $\mathrm{NaBH} 4, \mathrm{H} 2 \mathrm{O} / \mathrm{THF}$ (9:1), $25 \mathrm{oC}, 1.5 \mathrm{~h}$ & $\begin{array}{l}\text { hydrogenation of nitroarenes to aniline } \\
\text { derivatives }\end{array}$ & 19 \\
\hline PIS-Pd & K2CO3, H2O/DMF, 100 oC, 3 h & Suzuki-Miyaura cross-coupling reactions & 22 \\
\hline $\begin{array}{l}\text { Fe304@PNP1 and Fe304@ } \\
\text { PNP2 }\end{array}$ & $25 \mathrm{oC}$ in $\mathrm{H} 2 \mathrm{O}$-EtOH mixture & Suzuki-Miyaura cross-coupling reactions & 23 \\
\hline NHC-Pd polymer & [K4Fe(CN)6], DMF, K2CO3, 95 oC, $12 \mathrm{~h}$ & cyanation of various aryl halides & 24 \\
\hline Pd@CPP-C and Pd@CPP-Y & $25 \mathrm{oC}, 2 \mathrm{~h}, 1 \mathrm{~atm}$ of $\mathrm{H} 2$ & hydrogenation of olefins to alkanes & 25 \\
\hline CPP-1 and CPP-2 & $25 \mathrm{oC}, 1 \mathrm{~h}, 1 \mathrm{~atm}$ of $\mathrm{H} 2$ in ethanol medium & hydrogenation of nitroarenes & 26 \\
\hline $\mathrm{Ru}-\mathrm{A}, \mathrm{Ru}-\mathrm{B}$ and $\mathrm{Ru}-\mathrm{C}$ & NaBH4, THF/H2O (1:3), 25 oC & catalytic transfer hydrogenation of nitroarene & 27 \\
\hline Mn-POP & Isobutyraldehyde, $\mathrm{O} 2$ & olefin epoxidation & 28 \\
\hline $\begin{array}{l}\text { Mn@DVTA-1, Mn@DVTA-2 } \\
\text { and Mn@DVTA-3 }\end{array}$ & TBHP, 1 atm 02, 80 oC & $\begin{array}{l}\text { aerobic sp3 C-H bond oxidation of aromatic } \\
\text { hydrocarbons and alcohols }\end{array}$ & 29 \\
\hline PDVB-SB-Cu & Cs2C03, 115 oC, $16 \mathrm{~h}$ & $\begin{array}{l}\text { Ullmann biaryl ether coupling of iodobenzene } \\
\text { and phenol }\end{array}$ & 30 \\
\hline $\mathrm{Cu}-\mathrm{A}, \mathrm{Cu}-\mathrm{B}$ & $\mathrm{Cs} 2 \mathrm{CO} 3,110 \mathrm{oC}$ & $\begin{array}{l}\text { Ullmann coupling of aryl halides with primary } \\
\text { and secondary amines }\end{array}$ & 31 \\
\hline SPPOP-1, SPPOP-2, SPPOP-3 & $25 \mathrm{oC}$ & Esteriifcation and transesterification reactions & 32 \\
\hline HMP-1-SO3H & $25 \mathrm{oC}$ & Esteriifcation and transesterification reactions & 33 \\
\hline SPPTPA-1 & Microwave (140 oC), 20 min & conversion of carbohydrates to HMF & 34 \\
\hline
\end{tabular}

crystallographic facets in imine production using $1 \mathrm{~atm}$ pressure of molecular oxygen. This DVTA-POP based catalyst possess extraordinary thermal and chemical stability providing accessible coordinating sites for uptake of metal ions or metal nano-particles thereby making them an attractive candidate as new ideal platform in heterogeneous catalysis community. The synthesis of an efficient and stable $\mathrm{Cu}$-incorporated porous Schiff base-modified polydivinylbenzene (PDVB-SB-Cu) has been reported which was obtained by performing multiple steps reactions include nitration of benzene ring, reduction of nitro group, Schiff-base condensation, and followed by coordination with $\mathrm{Cu}(\mathrm{OAc})_{2}$ preserving its porous framework [30]. The heterogeneous PDVB-SB-Cu catalyst exhibited high catalytic activity in the Ullmann biaryl ether coupling of iodobenzene and phenol similar to that of the homogeneous Schiff base-stabilized copper catalyst with remarkable recyclability. Zhao, et al. have newly developed heteroge- 
neous $\mathrm{Cu}^{\circ} \mathrm{NPs}$ supported on porous organic polymer $(\mathrm{Cu}-\mathrm{B})$ catalyst for carrying out Ullmann coupling of aryl halides with primary and secondary amines in aqueous solution [31]. Cu-B showed higher catalytic performance compared with $\mathrm{Cu}-\mathrm{A}$ catalyst which was synthesized by chelate-assisted co-assembly strategy using $\mathrm{Cu}(\mathrm{acac})_{2}$, soluble phenol formaldehyde resin and poly (ethylene oxide)-block-poly (propylene oxide)-block-poly(ethylene oxide) triblock copolymer Pluronic F127 due to higher pore volume which is advantageous for access of reactants, and the hierarchical porosity is also favourable for easy mass transfer of reactants and products. The versatile substrate scope of this catalytic system with high product yield, high selectivity, and high mechanical stability of the polymer under the harsh reaction condition with no leaching of catalytic active centers proved this system very attractive in nature. Porous Organic Polymer was employed as a potential heterogeneous solid metal free organocatalyst which was developed with the introduction of active functional groups in the polymer framework by post-synthetic modification or precisely adjusting polymer matrix by selection of monomer. Recently, Bhaumik's group have rationally designed a series of new microporous organic polymers through FriedelCrafts alkylation reactions and one-step oxidative polymerization of triphenylamine in presence of anhydrous $\mathrm{FeCl}_{3}$. FriedelCrafts alkylation reaction between pyrene and bis(1,4-dibromomethyl)benzene at their different molar ratios in presence of $\mathrm{FeCl}_{3}$ has been adopted to synthesize a series of POPs namely PPOP-1, PPOP-2, and PPOP-3 [32]. All these pyrene based POP materials provide BET surface areas 615,570 and $508 \mathrm{~m}^{2} \mathrm{~g}^{-1}$, respectively. Same strategy was again followed for synthesis of a new hypercrosslinked supermicroporous polymer (HMP-1) with a BET surface area of $913 \mathrm{~m}^{2} \mathrm{~g}^{-1}$ via $\mathrm{FeCl}_{3}$ assisted Friedel-Crafts alkylation of carbazole and $\alpha, \alpha^{\prime}$-dibromo- $p$-xylene [33]. Nanoporous polytriphenylamine (PPTPA-1) polymer with exceptionally high surface area about $1437 \mathrm{~m}^{2} \mathrm{~g}^{-1}$ has been synthesized by onestep oxidative polymerization of triphenyl amine in presence of anhydrous $\mathrm{FeCl}_{3}$ [34]. All these microporous organic polymers were further functionalized with $-\mathrm{SO}_{3} \mathrm{H}$ groups to produce highly efficient acidic heterogeneous organocatalysts by reaction with a strong oxidizing reagent, $\mathrm{ClSO}_{3} \mathrm{H}$. Temperature programmed desorption of $\mathrm{NH}_{3}\left(\mathrm{NH}_{3}\right.$-TPD) measurement signifies high loading of $-\mathrm{SO}_{3} \mathrm{H}$ group in the porous framework. The organocatalysts exhibited high catalytic performance in production of biofuel and HMF. The performances of these solid sulfonated porous polymers PPOP acid catalysts were evaluated in the context of biodiesel synthesis via esterification/transesterification of long chain fatty acids/esters at room temperature. Similarly transesterification reaction of long chain fatty acids with methanol for biodiesel production with the corresponding product yield $93-99 \%$ was performed employing HMP-1- $\mathrm{SO}_{3} \mathrm{H}$ carbocatalyst. On the other hand, sulfonic acid functionalized PPTPA-1 (SPPTPA-1) catalyst was employed in catalytic conversion of carbohydrates to HMF which is a key intermediate of many industrially important chemicals and liquid fuels. All these aforementioned solid POP based organocatalysts exhibit outstanding recyclability and stability in the reaction conditions thereby offering useful alternatives suppressing the use of toxic homogeneous Lewis acid catalysts.
In this article, in a nutshell we have discussed the employment of porous organic polymers and various metal or metal oxide nanoparticles fabricated in porous organic polymers as heterogeneous and also metal free organocatalyst in performing various catalytic reactions.

An increasing interest has been paid for the development of POPs with high density of functionalities for choosing an efficient platform for loading active metal or metal oxide NPs in their framework due to their high surface area, suitable pore volume, chemical and thermal stability, strong binding ability and high catalyst loading. All the heterogeneous catalysts become a promising candidate in coupling reactions, hydrogenation reactions and various acid catalyzed reactions for production of fine chemicals, value-added products in petroleum refining and chemical industry. The versatile nature of Porous Organic Polymers (POPs) will make them attractive candidate in heterogeneous catalysts community addressing all the profound challenges in near future. Therefore researchers are keener in finding some sustainable, easy and low-cost methodologies for large scale preparation of such Porous Organic Polymer based materials that can be easily channelized in the design and development of novel heterogeneous catalysts.

\section{Acknowledgement}

SKK wishes to thankfully acknowledge CSIR for his respective CSIR-SRF fellowship. AB wishes to thank DST, New Delhi for providing funding though DST-SERB and DST-UKIERI project research grants and DST Unit on Nanoscience for providing instrumental facilities at IACS. RS, BMR and JM thank to Department of Science and Technology, India for DST-INSPIRE Faculty Research project grant (GAP-0522) in CSIR-IICT, Hyderabad.

\section{References}

1. Zhang Y, Riduan SN. Functional porous organic polymers for heterogeneous catalysis. Chem Soc Rev. 2012;41:2083-2094.

2. Sun Q, Dai Z, Meng X, Wang L, Xiao FS. Task-specific design of porous polymer heterogeneous catalysts beyond homogeneous counterparts. ACS Catal. 2015;5:4556-4567.

3. White RJ, Luque R, Budarin VL, Clark JH, Macquarrie DJ. Supported metal nanoparticles on porous materials. Methods and applications. Chem Soc Rev. 2009;38:481-494.

4. Mondal J, Gomes R, Modak A, Bhaumik A. Pd-anchored functionalized mesoporous materials as robust and recyclable heterogeneous catalysts for a series of C-C bond forming reactions. Recyclable Catalysis. 2013;10-33. doi: 10.2478/recat-2013-0001.

5. Dhakshinamoorthy A, Garcia H. Catalysis by metal nanoparticles embedded on metal-organic frameworks. Chem Soc Rev. 2012;41:52625284.

6. Dawson R, Cooper AI, Adams DJ. Nanoporous organic polymer networks. Progress in Polymer Science. 2012;37:530-563.

7. Zou X, Rena H, Zhu G. Topology-directed design of porous organic frameworks and their advanced applications. Chem Commun. 2013;49:3925-3936.

8. Suresh VM, Bonakala S, Atreya HS, Balasubramanian S, Maji TK. Amide Functionalized Microporous Organic Polymer (Am-MOP) for Selective 
$\mathrm{CO}_{2}$ Sorption and Catalysis. ACS Appl Mater Interfaces. 2014;6:46304637.

9. Fang Q, Gu S, Zheng J, Zhuang Z, Qiu S, Yan Y. 3D Microporous basefunctionalized covalent organic frameworks for size-selective catalysis. Angew Chem Int Ed. 2014;53:2878-2882.

10. Zhu Q-L, Li J, Xu Q. Immobilizing Metal Nanoparticles to Metal-Organic Frameworks with Size and Location Control for Optimizing Catalytic Performance. J Am Chem Soc. 2013;135:10210-10213.

11. Lu G, Li S, Guo Z, Farha OK, Hauser BG, Qi X, Wang Y, et al. Imparting Functionality To A Metal-Organic Framework Material By Controlled Nanoparticle Encapsulation. Nat Chem. 2012;4(4):310-6. doi: 10.1038/nchem.1272.

12. Cooper AI. Conjugated Microporous Polymers. Adv Mater. 2009;21:1291-1295.

13. Sun L, Liang Z, Yu J, Xu R. Luminescent microporous organic polymers containing the 1,3,5-tri(4-ethenylphenyl)benzene unit constructed by Heck coupling reaction. Polym Chem. 2013;4:1932-1938.

14. Zhang P, Weng Z, Guo J, Wang C. Solution-dispersible, colloidal, conjugated porous polymer networks with entrapped palladium nanocrystals for heterogeneous catalysis of the Suzuki-Miyaura coupling reaction. Chem Mater. 2011;23(23):5243-5249.

15. Pandey P, Farha OK, Spokoyny AM, Mirkin CA, Kanatzidis MG, Hupp JT, et al. A "click-based" porous organic polymer from tetrahedral building blocks. J Mater Chem. 2011;21:1700-1703.

16. Modak A, Mondal J, Bhaumik A. Highly porous organic polymer containing free $-\mathrm{CO}_{2} \mathrm{H}$ groups: a convenient carbocatalyst for indole $\mathrm{C}-\mathrm{H}$ activation at room temperature. ChemCatChem. 2013;5:1749-1753.

17. Gomes R, Bhanja P, Bhaumik A. Sulfonated porous organic polymer as a highly efficient catalyst for the synthesis of biodiesel at room temperature. Journal of Molecular Catalysis A Chemical. 2016;411:110116.

18. Zhou Y, Xiang Z, Cao D, Liu C-J. Covalent organic polymer supported palladium catalysts for CO oxidation. Chem. Commun. 2013;49(50):5633-5635.

19. Zhao H, Wang Y, Wang R. In situ formation of well-dispersed palladium nanoparticles immobilized in imidazolium-based organic ionic polymers. Chem. Commun. 2014;50:10871-10874.

20. Hasell T, Wood CD, Clowes R, Jones TA, Khimyak YZ, Adams DJ, et al. Palladium nanoparticle incorporation in conjugated microporous polymers by supercritical fluid processing. Chem Mater. 2010;22:557-564

21. Verde-Sesto E, Pintado-Sierra M, Corma A, Maya EM, de La Campa JG, Iglesias $\mathrm{M}$, et al. First pre-functionalised polymeric aromatic framework from mononitrotetrakis(iodophenyl)methane and its applications. Chem Eur J. 2014;20(17):5111-5120.
22. Zhao H, Li L, Wang Y, Wang R. Shape-controllable formation of polyimidazolium salts for stable palladium $\mathrm{N}$-heterocyclic carbene polymers. Sci Rep. 2014;4:5478. doi:10.1038/srep05478.

23. Zhao H, Li L, Wang J, Wang R. Spherical core-shell magnetic particles constructed by main-chain palladium $\mathrm{N}$-heterocyclic carbenes. Nanoscale. 2015;7(8):3532-3538.

24. Karimi B, Vafaeezadeh M, Akhavan PF. N-heterocyclic carbene-Pd polymers as reusable precatalysts for cyanation and Ullmann homocoupling of aryl halides: the role of solvent in product distribution. ChemCatChem. 2015;7(14):2248-2254.

25. Li L, Zhao H, Wang R. Tailorable synthesis of porous organic polymers decorating ultrafine palladium nanoparticles for hydrogenation of olefins. ACS Catal. 2015;5(2):948-955.

26. Li L, Zhao H, Wang J, Wang R. Facile fabrication of ultrafine palladium nanoparticles with size- and location-control in click-based porous organic polymers. ACS Nano. 2014;8(5):5352-5364.

27. Mondal J, Kundu SK, Ng WKH, Singuru R, Borah P, Hirao H, et al. Fabrication of ruthenium nanoparticles in porous organic polymers: towards advanced heterogeneous catalytic nanoreactors. Chem Eur J. 2015; 21(52): 19016-19027.

28. Liu T-T, Lin Z-J, Shi P-C, Ma T, Huang Y-B, Cao R. A Metallosalenbased porous organic polymer for olefin epoxidation. ChemCatChem 2015;7(15):2340-2345.

29. Mondal J, Borah P, Sreejith S, Nguyen KT, Han X, Ma X, et al. Morphology-tuned exceptional catalytic activity of porous-polymer-supported $\mathrm{Mn}_{3} \mathrm{O}_{4}$ in aerobic $\mathrm{sp}^{3} \mathrm{C}-\mathrm{H}$ bond oxidation of aromatic hydrocarbons and alcohols. ChemCatChem. 2014;6(12):3518-3529.

30. Wang L, Zhang J, Sun J, Zhu L, Zhang H, Liu F, et al. Copper-incorporated porous polydivinylbenzene as efficient and recyclable heterogeneous catalyst in Ullmann biaryl ether coupling. ChemCatChem. 2013;5:(6): 1606-1613.

31. Mondal J, Biswas A, Chiba S, Zhao Y. $\mathrm{Cu}^{0}$ nanoparticles deposited on nanoporous polymers: a recyclable heterogeneous nanocatalyst for Ullmann coupling of aryl halides with amines in water. Sci Rep. 2015;5:8294; doi:10.1038/srep08294.

32. Kundu SK, Bhaumik A. Pyrene-based porous organic polymers as efficient catalytic support for the synthesis of biodiesels at room temperature. ACS Sustainable Chem Eng. 2015;3(8):1715-1723.

33. Bhunia S, Banerjee B, Bhaumik A. A new hypercrosslinked supermicroporous polymer, scope for sulfonation, and its catalytic potential for the efficient synthesis of biodiesel at room temperature. Chem Commun. 2015; 51:5020-5023.

34. Mondal S, Mondal J, Bhaumik A. Sulfonated porous polymeric nanofibers as an efficient solid acid catalyst for the production of 5-hydroxymethylfurfural from biomass. ChemCatChem 2015;7:3570-3578. 


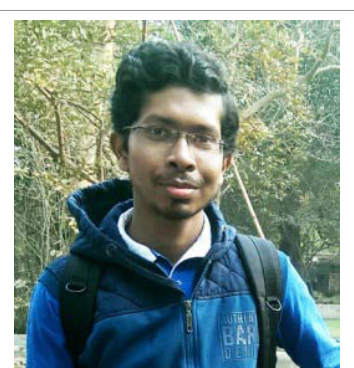

Sudipta KK, was born in Hooghly (India) in 1988. He is working as a senior research fellow at Indian Association for the Cultivation of Science, India under the supervision of Prof. Asim Bhaumik since 2011. He received his B.Sc. degree in Chemistry from the Raja Peary Mohan College, Uttarpara, University of Calcutta in 2009 and M.Sc degree from Ramakrishna Mission Residential College, Narendrapur under University of Calcutta in 2011. He has co-authored over 10 scientific publications. The main objectives of his work are the synthesis of microporous organic polymers and the exploration of their emerging application in the field of heterogeneous catalysis and $\mathrm{CO}_{2}$ and $\mathrm{H}_{2}$ storage applications.

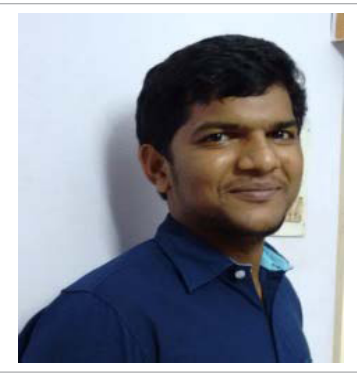

Ramana S, was born in Srikakulam (India) in 1989. He is working as a junior research fellow at Indian Institute of Chemical Technology Hyderabad, India under the supervision of Dr. B. Mahipal Reddy since 2014. He received his B.Sc. degree in Chemistry from the A.B.N \& P.R.R College of science, Kovvur, Andhra University in 2010 and M.Sc degree from Pondicherry University in 2012. His research interests include Design and development of nano sized ceria based mixed oxides and the exploration of their emerging application in the field of heterogeneous catalysis.

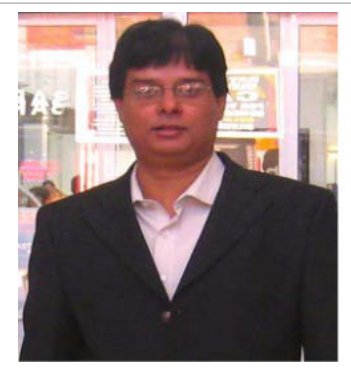

Dr Bhaumik A, received his Ph.D in Chemistry from National Chemical Laboratory, Pune in 1997. Then he moved at The University of Tokyo as JSPS fellow (1997-1999) and at Toyota CRDL, Japan (1999-2001) for post-doctoral studies. After finishing post-doctoral research studies in 2001 he had joined in the Department of Materials Science of Indian Association for the Cultivation of Science as a faculty. At present he is a full Professor there. His current research includes designing functionalized porous materials for adsorption, gas storage, catalysis, sensing, optoelectronics and drug delivery. He has published about 300 peer-reviewed research articles in International journals. He serves on editorial boards or advisory boards of several journals including Green Chemistry, Green and Sustainable Chemistry, Recent Patents on Catalysis, Recyclable Catalysts, Advances in Chemical Engineering, The Open Catalysis Journal and a Fellow of The Royal Society of Chemistry.

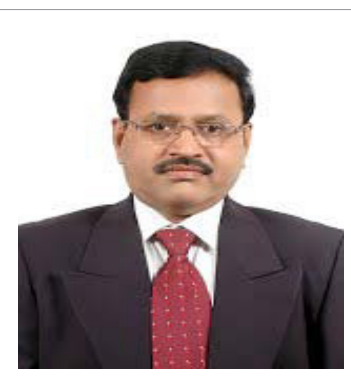

Dr Benjaram MR, is a versatile chemist with varied experience in catalysis, physical chemistry, organic chemistry, and materials science with special expertise in the areas of nanomaterials and green technologies. He started his scientific research career in the year 1984 at the CSIR-Indian Institute of Chemical Technology, Hyderabad, where he holds a Chief Scientist and Head of Inorganic and Physical Chemistry division position. He has been a visiting scientist or an award fellow at the Institute of Catalysis, Novosibrisk, Russia (1986-87), Institute of Physical Chemistry, LMU, Munich, Germany (1988-89 and 1998-99), State University of New York, Buffalo, USA (1993-94), Texas A\&M University, College Station, USA (1994-95), AIST, Osaka, Japan (2001), Institute for Research on Catalysis, Lyon, France (2001, 2003, and 2005), Inha University, Incheon, South Korea (2007-08), Ruhr University Bochum, Germany (2005, 2010, and 2012), Paul Scherrer Institute, Villigen, Switzerland (2013 and 2014), and RMIT University, Melbourne, Australia (2013). Prof Reddy is the recipient of prestigious CSIR Young Scientist Award in Chemical Sciences (1990), Catalysis Society of India (CSI) Young Scientist Award, and Associate Fellowship of Third World Academy of Sciences, in addition to several other awards, fellowships and honours. Prof Reddy is an elected fellow of the Indian National Academy of Engineering (FNAE), the National Academy of Sciences, India (FNASc), Royal Society of Chemistry (FRSC), the Andhra Pradesh Academy of Sciences (FAPASc), and the Telangana Academy of Sciences (FTAS).

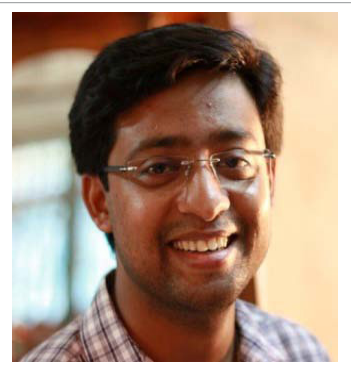

Dr John Mondal, joined Inorganic and Physical Chemistry Division at CSIR-Indian Institute of Chemical Technology (IICT), Hyderabad as a tenure-track Assistant Professor in 2015 after receiving Ph.D in Chemistry from Indian Association for the Cultivation of Science, Kolkata in 2013 followed by a postdoctoral fellowship at Nanyang Technological University, Singapore (2013-2015) with Prof. Yanli Zhao. He is currently leading a research group interested in Nanocatalysis, Magnetically recoverable bimetallic nanocatalyst, Heterogeneous Catalysis and Nano-structured materials. The present thrust of his research is the development of Novel Heterogeneous Catalysts with the fusion of metal or metal oxide NPs and Porous Organic Polymers (POPs) and to perform several catalytic transformations in Biofuel upgrade, Bio-mass conversion, Photocatalytic Hydrogen generation from Bio-glycerol. Another objective of his research is to explore Shape-dependent and Size-dependent catalytic activity difference and to merge them with computational DFT calculation studies. 